\title{
Heike Ortner
}

\section{,Therapeutischer' Widerspruch: Epistemische Rechte und Pflichten in physiotherapeutischen Interaktionen}

\begin{abstract}
In einer Physiotherapie im Rahmen einer neurorehabilitativen Maßnahme wird verbal und körperlich fortlaufend teils explizit und teils implizit die körperliche Bewegungsfähigkeit und -ausführung evaluiert. Der Beitrag fokussiert die konversationelle Aushandlung von epistemischer Autorität und teilweise deontischer Autorität anhand des Beispiels einer spezifischen Praktik: des therapeutischen Widersprechens im Sinne einer Bewertung, die von den Selbstbewertungen (oft Selbstabwertungen) der Patient*innen abweicht. Die Therapeut*innen sind dabei aufgrund ihres Fachwissens und ihrer Berufserfahrung, aber auch aufgrund von konversationellen Normen der zwischenmenschlichen Beziehung gefordert, mit den Patient*innen unter Einsatz unterschiedlicher verbaler und multimodaler Mittel alternative Perspektivierungen interaktiv auszuhandeln.
\end{abstract}

Keywords: Physiotherapie, Rehabilitation, Widersprechen, Bewerten, Multimodalität

\section{Einleitung}

Ein Aufenthalt in einem neurologischen Rehabilitationszentrum soll Patientinnen und Patienten dabei helfen, individuell zugeschnittene Ziele $\mathrm{zu}$ erreichen (vgl. Frommelt \& Grötzbach 2010: 13). Die junge Patientin aus Beispiel $1^{1}$ befindet sich zum Zeitpunkt der Ton- und Videoaufnahme zum wiederholten Mal in einer Rehabilitationsklinik und kennt den Physiotherapeuten gut. In dem fol-

1 Die Transkription orientiert sich an GAT 2 (Selting et al. 2009), ergänzt durch die bei Mondada (2014) veranschaulichten Konventionen zur Notation verschiedener Ebenen interaktionaler Displays - leicht angepasst, um dem Material des vorliegenden Beitrags besser gerecht zu werden. Eine kurze Lesehilfe: Die fett hervorgehobene Zeile enthält das verbale Transkript mit zeitlich zugeordneten Einsatz- $\left({ }^{\star}\right)$ und Endpunkten $\left(/{ }^{\star}\right)$ für multimodale Displays. Die kursive Zeile stellt eine am Standarddeutschen orientierte Übertragung dar. Darunter finden sich verbale Umschreibungen der verkörperten Interaktionsanteile ${ }^{\star}=$ Gestik, $\mu=$ therapeutische Handlungen, $+=$ Blickverhalten, $¥$ = andere Handlungen, $\Delta$ = Körperorientierung).

Heike Ortner, Universität Innsbruck, Institut für Germanistik, heike.ortner@uibk.ac.at.

Ә Open Access. ( 2021 Heike Ortner, publiziert von De Gruyter. (c) BY-NC-ND Dieses Werk ist lizenziert unter einer Creative Commons Namensnennung - Nicht-kommerziell - Keine Bearbeitung 4.0 International Lizenz. https://doi.org/10.1515/9783110688696-009 
genden kurzen Ausschnitt widerspricht er einer negativen Einschätzung der Patientin in Bezug auf die Leistung des Musculus psoas major.

Beispiel 1: Der is schwach bei mir

Einheit 001, ((00:02:40.682 - 00:03:11.800))

$\mathrm{T} 1$ = Therapeut, $\mathrm{P} 1$ = Patientin

01 T1 $>*>+>\mu>¥_{\mathrm{p} 1}$ des $/ * ¥_{\mathrm{t} 1}$ is OAna von die kräftigschten /Y mUskel $¥$ wos ma HOBM;

das ist einer der kräftigsten Muskeln was wir haben

t1 >* kratzt sich am Kinn /*

$>+$ blickt auf LL und Hüfte von $p 1$

$>\mu$ hält mit LH LL von p1 fest

$¥$ richtet Rolle unter Knien von p1 zurecht / $¥$

$¥$ kniet

sich auf Liege

p1 >¥ liegt in Rückenlage mit angezogenen Knien auf der Liege-->>

$02 \mathrm{~T} 1 \quad(0.5) \mathrm{der}$

$03 \quad(0.7) / ¥(0.3)$

t1 ------- $¥$

04 P1 ((lacht)) der $\mu$ is * SCHWACH $¥+$ bei mir. der ist schwach bei mir

$\mu$ positioniert und manipuliert mit BH LL von p1 ----->>

* berührt/kratzt sich mit LH am Nacken -----------> $¥$ hebt Kopf

$05 \quad$ T1 $<$ rall > na $¥[$ des $]>/ ¥$

nein das

$¥$ beugt sich weiter nach vorn $/ ¥$

06

p1

$>*$

$\langle<:-)>[$ das ] is der SCHWÄCH+*ste $>$ bei mir. /*

das ist der schwächste bei mir

p1

p1

t1

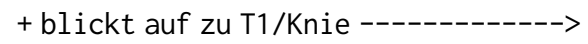

07 T1 der * der springt /* schneller an wie $\mu$ ANdere;

der der springt schneller an wie andere

p1 * lässt LH auf die Matte fallen /*

t1

$\mu$ positioniert und manipuliert mit RH Knie, mit LH 1 . Hüfte von p1 ---------------------->> 
Der Therapeut unterbricht die schon seit einer Weile nicht funktionierende Entspannung des genannten Muskels, um sich in Z. 03 auf der Liege gegenüber der Patientin neu zu positionieren und die stützende Rolle zurechtzurücken. In Z. 04 reagiert die Patientin mit einem Lachen (begleitet von einer Selbstberührung), gefolgt von einer negativen Beurteilung ihres Muskels, die sie in Z. 06 trotz des in der Überlappung von Z. 05 angedeuteten Widerspruchs des Therapeuten mit einer hyperbolischen Abwertung verstärkt, unterstützt durch das Suchen nach Blickkontakt. Obwohl das Lachen und die ,smile voice“ auf scherzhafte Modalität hindeuten, ist die Selbstabwertung im Sinne eines krankheitsbedingten Defizits ernst gemeint und wird vom Therapeuten mit einer Erläuterung des Problems als nicht von der Kraft abhängig relativiert. Multimodal unterstützt er dies, indem er einen neuen Anlauf startet, die angestrebte Entspannung des Muskels zu erleichtern. Durch das therapeutische Handeln sind sowohl das Blickverhalten als auch gestische Displays eingeschränkt.

Das Fachwissen, das der Therapeut über den fokussierten Muskel hat, und seine Interpretation des Verhaltens infolge der therapeutischen Manipulation stehen hier im Widerspruch zur verbal und körperlich explizierten Selbstabwertung der Patientin. Mit seiner Meinungsäußerung wird der Therapeut einerseits der interaktionalen Anforderung gerecht, dass die negative Selbstbewertung in Z. 04 und Z. 06 eine Reaktion konditionell relevant setzt (im Sinne von Schegloff 1972), andererseits beantwortet er die mit dem Suchen nach Blickkontakt nonverbal gestellte Frage der Patientin nach seiner professionellen Meinung als Experte. Anders als in Alltagsgesprächen ist es im Kontext einer Physiotherapie nicht nur möglich, sondern in vielen Fällen erforderlich, realistische und somit auch negative Bewertungen körperlicher Funktionen abzugeben. Dies steht dem Therapeuten aufgrund seines epistemischen Status als Experte zu (aufgrund seiner „knowledgeability“, nach Heritage 2012). Seinem patientenzentrierten Rollenverständnis entsprechend begründet er seine Einschätzung, die in eine über den Transkriptausschnitt hinausgehende längere Erklärung der Funktion des Psoas und der noch erforderlichen Therapiemaßnahmen mündet.

Im Rahmen des Pilotprojekts ${ }^{2}$ „Therapeutische Bewegungsinstruktionen in der Neurorehabilitation“ wurden physiotherapeutische Interaktionen in einer österreichischen Reha-Klinik videografiert und unter verschiedenen Gesichtspunkten nach Kriterien der multimodalen Analyse von natürlichen Interaktio-

2 Die Erhebung wurde von der zuständigen Ethikkommission mit strengen Auflagen genehmigt. Dazu gehört, dass in Publikationen keine Abbildungen verwendet werden dürfen. Aus Datenschutzgründen werden zu Alter, Herkunft und Diagnose der beteiligten Personen nur vage Angaben gemacht, insoweit es für das Verständnis des Beispiels notwendig ist. 
nen (vgl. z. B. Deppermann 2013) ausgewertet. Der vorliegende Beitrag legt den Fokus auf eine spezifische professionelle Praktik: Therapeutenseitiges Widersprechen wird exemplarischen multimodalen Detailanalysen unterzogen und einzelfallübergreifend kategorisiert. Die übergeordneten Forschungsfragen lauten: Wie, mit welchen multimodalen Ressourcen und mit welchen therapeutischen Funktionen wird Nicht-Übereinstimmen ko-konstruiert bzw. ein Konsens über therapeutische Maßnahmen ausgehandelt?

Das Korpus umfasst insgesamt 420 Minuten Video- und Tonmaterial aus zehn Einheiten mit neun verschiedenen Therapeut*innen und Patient*innen. Methodische Grundlage der Analyse ist die qualitative multimodale Interaktionsanalyse bzw. die Analyse ,verkörperter Interaktion“ (embodied interaction, Mondada 2013), die alle Ausdrucksressourcen - Stimme, Mimik, Gestik, Körperposition, Blickverhalten, Berührungen, Bewegungen im Raum, Nähe- und Distanzverhalten, Objektmanipulation - als einerseits sequenziell strukturiert, andererseits komplex miteinander koordiniert betrachtet (vgl. Beiträge in Schmitt 2007).

\section{Grundlagen der Analyse}

Die linguistische Physiotherapieforschung ist vor allem von konversationsanalytischen Ansätzen geprägt und entsteht häufig aus einem unmittelbaren Praxiskontext, in dem typische sprachliche Praktiken in verschiedenen Phasen einer physiotherapeutischen Interaktion evaluiert werden, z. B. die Aushandlung der Ziele der Therapie (u. a. Parry 2004a). Vereinfacht gesagt beruht das motorische (Neu-)Lernen in der Physiotherapie auf der Umsetzung verbaler und körperlicher Anleitungen mit manueller Unterstützung, was fortgesetzte Beobachtung, Beurteilung und Bearbeitung von normabweichenden Bewegungen sowie ständige Kooperationsbereitschaft der Patient`innen erfordert (vgl. Parry 2004b: 978). Eine physiotherapeutische Einheit wird von Interaktionsrollen (Status- und Rollenüberzeugungen) und Identitätskonstruktionen der beteiligten Personen geprägt, aber auch von institutionellen Bedingungen und situativen Umständen (z. B. Therapiefortschritt und Tageszeit).

Der vorliegende Beitrag fokussiert die konversationelle Aushandlung von epistemischer Autorität (beruhend auf Wissen über Sachverhalte, vgl. Heritage \& Raymond 2005) und teilweise deontischer Autorität (beruhend auf situativen Vorrechten, vgl. Stevanovic \& Peräkylä 2012). In den folgenden Ausführungen werden drei Aspekte dieses Themas theoretisch verortet: Epistemische Autorität im medizinischen Kontext, Bewerten als therapeutische Aufgabe sowie Wider- 
spruch gegen Bewertungen als konversationelle Praktik im Zusammenhang mit der Präferenzorganisation.

Heritage (2012: 4) unterscheidet zwischen epistemic stance, dem Ausdruck der Haltung zum Wahrheitsgehalt von Aussagen, und epistemic status, d. h. der in der Einleitung bereits erwähnten „knowledgeability“ relativ zu anderen Partizipierenden. Meist wird dieser Aspekt in sozialen Interaktionen implizit vorausgesetzt (vgl. Stevanovic \& Peräkylä 2012: 317). Ob auf ein Display epistemischer und deontischer Autorität kongruente oder inkongruente Reaktionen erfolgen, ist sehr stark abhängig vom common ground in Hinblick auf die Verantwortung der Partizipierenden für das in ihren Äußerungen konstruierte Wissen (Heritage \& Raymond 2005). Die Wissensasymmetrie zwischen den Therapeut*innen als Expert^innen und den Patient*innen als medizinischen Laien und gleichzeitig Expert*innen für ihre individuellen Beschwerden ist prinzipiell funktional (vgl. Parry 2004a: 678). Die Therapeut*innen verfügen nicht nur über breites Fachwissen, sondern folgen in der Interaktion auch den in der Ausbildung erworbenen normativen Modellen und (Quasi-)Theorien ihres Berufs, den „professional stocks of interactional knowledge“ (Peräkylä \& Vehviläinen 2003: 730). Sehr allgemeine Empfehlungen für das richtige Feedback in der Physiotherapie sind externe Rückmeldungen (Attributionen von Problemen auf nicht persönliche sowie auf veränderbare Ursachen), positive Verstärkung mit Lob, mehrfache Wiederholung einer Übung und genügend Pausen (vgl. Fries \& Freivogel 2010: 245).

Ein Teil der therapeutischen Aufgaben sind nun wie erwähnt fortlaufende Bewertungen der Bewegungsausführungen. In der Gesprächsanalyse wurden Bewertungen insbesondere unter dem Blickwinkel ihrer sequenziellen Organisation, also ihrer Positionierung und der darauf folgenden Gesprächsschritte thematisiert, vor allem in Zusammenhang mit der Präferenzorganisation (vgl. Pomerantz 1984; Auer \& Uhmann 1982; Levinson 2000). Im vorliegenden Beitrag wird ein breiteres Verständnis angelegt: Als Bewertung gilt alles, was mit Termini wie „modality, evaluation, attitude, affect, subjectivity, or stance“ (Kärkkäinen 2006: 700), mit ,appraisal‘ aus der systemisch-funktionalen Linguistik nach Halliday (systematisiert von Martin \& White 2005) oder in Anlehnung an Goodwin \& Goodwin (1992), Kärkkäinen (2006) und Heritage (2012) als ,assessment' bezeichnet wird. Bewertungen können sich körperlich oder durch eine grammatische oder lexikalische Wahl eines Interaktanten ausdrücken. In der interaktionalen Forschung wird dies üblicherweise von der dynamischen intersubjektiven Konstruktion von Positionierung (stance) unterschieden. Stance wird beispielsweise analytisch rekonstruierbar durch körperliche, syntaktische, semantische und/oder prosodische Parallelität oder ,Resonanz' zwischen Interagierenden (vgl. Kärkkäinen 2006). Zentral ist für die folgenden Ausführungen die Sichtweise von Goodwin \& Goodwin (1992: 155-156), die verschiedene 
Bedeutungsebenen von assessments beschreiben, darunter assessment activities, bei denen Bewertungen von mehreren Partizipant^innen gemeinsam vorgenommen werden. Es handelt sich um shared understandings, die verschiedene interaktive Konsequenzen haben bzw. den Partizipant`innen Vorhersagen über die interaktive Struktur erlauben (vgl. Goodwin \& Goodwin 1992: 166-167).

In einer therapeutischen Interaktion gelten jedoch andere Regeln und Normen als in einem Alltagsgespräch, sodass nicht alle Erkenntnisse zu Bewertungen aus der konversationsanalytischen Forschung ohne Weiteres übertragbar sind. So sind Bewertungshandlungen (assessments) sehr häufig, aber zwischen den Partizipant`innen ungleich verteilt. Die Therapeut`innen sind ständig vor allem mithilfe ihres ,professionellen Blicks' (Goodwin 1994) und haptischer Wahrnehmung (Berührungen) mit Bewertungen beschäftigt, müssen ihre Einschätzungen jedoch nicht fortlaufend verbal oder auf andere Weise interaktional greifbar preisgeben, sondern nur an bestimmten Positionen während der manuellen Therapie oder wenn die Patient*innen Bewertungen einfordern. In dem von mir aufgezeichneten Material sind folgende drei Arten von patient*innenseitigen Bewertungsabfragen sehr häufig:

1. performance feedback (Bewertungen des aktuellen Therapiegeschehens und der aktuellen Ausführung einer Übung)

2. Bewertungen der Fortschritte, des bereits Erreichten

3. Zukunftsprognosen über den zu erwartenden weiteren Therapieerfolg oder individuelle Perspektiven

Gill (1998: 346) beschreibt ähnliche Prozesse in Arzt-Patienten-Gesprächen und dass in diesem Kontext die Patient ${ }^{\star}$ innen die Position für Nachfragen sowie die Demonstration ihres Wissens genau ausloten und unsicheres Wissen als Einladung zur Abgabe einer ärztlichen Expertise präsentieren. In Bezug auf eine Physiotherapie in der Neurorehabilitation bedeutet dies, dass die vorgenommenen Bewertungen eine sorgfältig getroffene Auswahl aus einem sehr breiten Repertoire darstellen: Dieses Repertoire betrifft nicht nur die lexikalischen und syntaktischen Bewertungsausdrücke und die multimodalen Optionen des Bewertens, sondern auch die Bewertungsqualität (z. B. Bevorzugung positiver Aspekte), die Bewertungstiefe (Exaktheit, Ausführlichkeit) und die Bewertungsintensität. Gegenstand des Beitrags sind jedoch nicht die Bewertungen an sich, sondern ihre interaktionalen Konsequenzen, und zwar konkret wenn sie einen Widerspruch gegen eine patient`innenseitig geäußerte Bewertung darstellen. Widerspruchssequenzen mit dem prototypischen Ablauf Behauptung, Vollzug des ,Widersprechens', Vollzug einer Bearbeitungsaktivität (z. B. Einwand) und Ratifikation erfolgreicher Bearbeitung wurden ausführlich von Spranz-Fogasy (1986) anhand 
des Beispiels ,Schlichtungsgespräche‘ dargestellt. Einige der von ihm genannten Formen und Techniken werden in der Diskussion der Beispiele aufgenommen.

Prinzipiell ist der Widerspruch gegenüber einer Beurteilung in Paarsequenzen weniger präferiert als Zustimmung (vgl. Levinson 2000: 365), mit zwei Ausnahmen: Bewertungen, die eine Selbstanklage darstellen, sodass Nicht-Widersprechen gesichtsverletzende Zustimmung bedeutet, sowie Komplimente (vgl. Pomerantz 1984; vgl. auch Schegloff 2007: 59-60). Widerspruch ist strukturell betrachtet also kein alignment (gemeinsame Ausrichtung), kann aber auch kooperativ sein und affiliation (Zugehörigkeit) vermitteln (vgl. Lee \& Tanaka 2016: 3-4). Heritage \& Raymond (2005: 16) betrachten das Management von wissensbasierten Rechten und Verantwortungen an verschiedenen konversationellen Positionen und stellen eine Verbindung zu Höflichkeit und positivem Gesicht (face) her. Je nach Position des assessments ergeben sich unterschiedliche epistemische Rechte.

Denken wir nun Präferenzorganisation, Bewerten, Widerspruch und epistemische Rechte zusammen, stellt sich die Frage, welche epistemischen Evaluationsrechte und -pflichten in einer Therapieinteraktion bestehen.

\section{Ergebnisse: Typen des ,therapeutischen Widersprechens ${ }^{*}$}

\subsection{Widersprechen als professionelles Recht}

Anders als in vielen Formen von Arzt-Patienten-Gesprächen, in denen institutionelle und emotionale Anforderungen der Patientenbetreuung in Konflikt treten (vgl. Ruusuvuori 2007: 598), gibt es in der Physiotherapie Zeit für das vertiefte Eingehen auf Probleme, und zwar ohne dass dies die Behandlung verzögert, da solche Sequenzen parallel zum körperlichen Handeln stattfinden können. In Beispiel 2 spricht die ältere Patientin, die nach einem Schlaganfall das freie Stehen und Gehen neu lernen muss, ein Problem an: eine unwillkürliche Pendelbewegung des rechten Fußes beim Aufsetzen.

Beispiel 2: Ein bisschen Sorgen

Einheit 004, ((12:03.903-12:22.360))

T5 = Physiotherapeutin, T6 = Ergotherapeutin, P4 = Patientin

((P4 sitzt auf der Liege, vor ihr steht ein Pult, auf dem sie ihre Hände abstützt. T5 (Physiotherapeutin) sitzt links von ihr auf einem Hocker, T6 (Ergotherapeutin) hockt rechts von ihr. Alle drei blicken auf P4s Füße.)) 
01 P4 mei (. )* mei $\uparrow F U A S S$ tuat $\Delta$ 0lleweil /* so / $\Delta$ somein mein Fuß tut immer wieder so so

p4

t5 * schüttelt leicht den Kopf /*

in Richtung von P4s Füßen $/ \Delta$

$<\mathrm{p}>$ des mocht $\Delta_{\mathrm{t} 6}$ ma eigentli $¥$ a bissi $>/ ¥$ SURgn . das macht mir eigentlich ein bisschen Sorgen

$¥$ rückt mit dem Stuhl etwas zurück / $¥$

03 T6 an LINken + oder an [RECHten]?

den linken oder den rechten?

t6

$04 \quad$ P4

$$
+ \text { blickt in P4s Gesicht }
$$

[na der] RECHte tuat olleweil so $()<.<$, len $>$ a bissi umanOnder'>

nein der rechte tut immer wieder so ein bisschen herum

05

$$
(0.3)+(0.2)
$$

$---->+$ blickt auf P4s Füße

06 T5 oba $i($.$) also ich \mu_{\mathrm{t} 5}(0.7)$ wenn I des $\mu_{\mathrm{t} 6}$ jetzt so ( - ) be[0B]ochtaber ich (.) also ich (0.7) wenn ich das jetzt so beobachte

r. Knöchel

$\mu$ stabilisiert P4s r. Knöchel->>

07 T5 hob i jetzt NIT des gfühl-

hab ich jetzt nicht das Gefühl

08 T5 dass es $\mu$ Irgendwie wEsentli $+_{t 5}$ SCHLECHter $+_{p 4}$ wordn isch $+_{p 4}$ [gö?] dass es irgendwie wesentlich schlechter geworden ist, gell?

und dann auf ihre Füße

+ blickt kurz in T5s Gesicht

09 P4 [hm hm] (-)

10 P4 $i-i$ * kunn des /* söba $i$ kunn des NIT so guat sogn $<<p p>>$ kimmt ma vor; >

ich kann das selbst ich kann das nicht so gut sagen kommt mir vor p4 * zuckt mit den Achseln /*

In Z.01 vermittelt die Patientin vor allem durch ihre veränderte Körperorientierung, dass irgendetwas mit ihrem Fuß nicht stimmt. Die Aufmerksamkeit der Therapeutinnen liegt bereits bei den Füßen, verbal vermittelt die Patientin in Z.01 und Z.04 nur sehr vage, was ihr Sorgen bereitet. Die Nachfrage von T6 in Z. 03 deutet bereits an, dass das angesprochene Problem nicht eindeutig ist. 
Nachdem sie durch ihr Blickverhalten eine Begutachtung vermittelt hat, gibt T5 in Z. 06 bis Z. 08 an, dass ihrer professionellen Einschätzung nach das angesprochene Problem schon länger besteht, sich aber nicht verändert hat. Sie erkennt also das unerwünschte unkontrollierbare Bewegungsmuster des Fußes an, widerspricht der Patientin jedoch hinsichtlich der Einordnung als akut und letztendlich auch der aktuellen Relevanz, sodass wieder zur eigentlichen Übung übergegangen werden kann. Diese Technik kann nach Spranz-Fogasy (1986: 40) als Alternativbehauptung eingestuft werden. Obwohl die Therapeutin mit dem Herstellen von Blickkontakt und einer einfühlsamen Sprechweise empathisches Eingehen auf die Problemdarstellung vermittelt, zieht sich die Patientin in $\mathrm{Z}$. 09-10 merklich zurück, was durch das Abwenden des Blickes, ein Achselzucken und ihre verbale Abschwächung der zuvor geäußerten Sorgen ausgedrückt wird.

So wie Beispiel 2 widerspricht die therapeutische Evaluation sehr häufig den subjektiven Einschätzungen der Patient*innen (vgl. auch ähnliche Ergebnisse in der Studie von Josephson et al. 2015: 131-134). Und wie in Z. 06 ist auch in den meisten anderen Instanzen dieser Art des Widerspruchs das von Heritage (2002) ausführlich beschriebene Phänomen des oh-Vorlaufs zu beobachten, also einer Einleitung des Nicht-Übereinstimmens mit einem Vorläuferelement insbesondere dann, wenn der/die widersprechende Partizipant ${ }^{\star}$ in epistemische Priorität für sich beanspruchen kann (vgl. Heritage 2002: 32).

Sequenziell und multimodal wird der Widerspruch häufig durch die Ankündigung der Uneinigkeit, z. B. mit einem Kopfschütteln, eingeleitet (ein sogenanntes „pre-disagreement“ nach Schegloff 2007: 69). Der eigentliche Widerspruch muss aber zwingend explizit und verbal ausgeführt werden. Wie im Beispiel wird dies aber erst nach einer Phase der verkörperten - manuellen oder visuellen Evaluation vorgenommen. Es bleibt jedoch nur selten dabei: Die Patient^innen deuten ihrerseits hauptsächlich durch multimodale Displays wie Senken des Blicks, Wegschauen und schwache Rezeptionssignale an, dass sie mit der professionellen Einschätzung nicht einverstanden sind oder weiteren Erklärungsbedarf haben (siehe Z. 08 und Z. 09). Die Therapeut*innen nehmen dies in den meisten Fällen wahr und führen ihre Einschätzung weiter aus: mit verbalen Erläuterungen, oft gestützt durch manuelle Veranschaulichungen. Typisch ist dabei auch die durch das Herstellen von Blickkontakt realisierte Vergewisserung, dass die Patient`innen die Erklärungen verstehen. Die Patient`innen ihrerseits bringen sich mit intensivierten Beurteilungen ihrer Schwierigkeiten ein, zeigen das Problem übertrieben vor, klagen verbal mit höherer Lautstärke und stärkeren Betonungen. Es handelt sich auf beiden Seiten um Grounding-Aktivitäten (nach Clark \& Brennan 1991; Deppermann \& Schmitt 2008). Typischerweise enden solche oft 
relativ langen Abschnitte jedoch damit, dass sich die therapeutenseitige Evaluation durchsetzt.

Das Nicht-Übereinstimmen ist in Fällen wie diesen insofern ein ,professionelles Recht“, als das troubles telling der Patient*innen interaktional bearbeitet werden muss. Die interaktive Konstruktion beschränkt sich nicht auf die sequenzielle Abfolge 1. Bewertung durch Patient*in - 2. Widerspruch von Therapeut*in mit alternativer Bewertung - 3. Quittierung durch Patient*in mit unterschiedlichen Exkursen und Schleifen. Die Dauer und Qualität der Sequenz ist ganz wesentlich von den gleichzeitig zur verbalen Ausführung ablaufenden multimodalen Displays beider Seiten abhängig. In Beispiel 2 etwa gesteht die Patientin zwar in Z. 09-10 zu, dass ihr im Gegensatz zur Therapeutin die Möglichkeit der klaren Einstufung fehlt, aber ihre körperliche Ausrichtung zum problembehafteten Fuß vermittelt, dass sie die epistemischen Rechte in Hinblick auf ihr subjektives Empfinden nicht abzugeben bereit ist. Dieser Rückzug auf das individuelle Körpergefühl ist seinerseits eine Technik des Widersprechens in physiotherapeutischen Interaktionen, die nur den Patient*innen als Möglichkeit offensteht.

\subsection{Widersprechen als professionelle Pflicht}

In Beispiel 1 zog die scherzhaft übertriebene Selbstabwertung der Patientin eine ruhige, aber eindeutige Gegenbewertung des Therapeuten nach sich. Ähnliches geschieht in Beispiel 3, in dem dieselbe Patientin wie in Beispiel 2 in derselben Phase der Einheit mit einer verbal und nonverbal vollzogenen starken Selbstabwertung ein Lob abwehrt, was eine entsprechende Verhandlung über das Geleistete, das Können und Nicht-Können der Patientin nach sich zieht.

\section{Beispiel 3: Nicht so einfach}

Einheit 004, ((11:26.902-11:51.666))

T.05 = Physiotherapeutin, T.06 = Ergotherapeutin, P.04 = Patientin

((P4 steht an dem aufgebauten Pult (siehe Bsp. 3) und hält sich daran mit den Händen fest. Rechts von ihr sitzt T5 auf einem Hocker, links von ihr steht T6, beide blicken auf P4s Torso.))

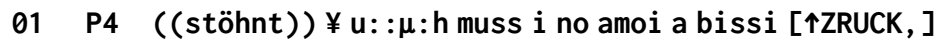
uh ich muss noch einmal ein bisschen zurück

p4

t5

$02 \quad$ T5
$¥$ wankt und setzt sich schnell auf die Liege zurück

$\mu$ fasst schnell zu und stützt $\mathrm{P} 4 \mathrm{mit} \mathrm{BH}$ an der Hüfte

[!PAU!se. ] 
03 T5 !BRA:! $\Delta \mathrm{vo},+$

bravo

p4 $\triangle$ richtet sich auf------------------------------------->

04 T6 SE/AHR + [guat; ]

sehr gut

p4 $\quad->/ \Delta$

t5 + blickt hinauf zum Spielbrett------------------------>

05 T5 [UND?]

und?

06 T6 die + NÄCHsten zwoa-a-hoib rEIhen;

die nächsten zweieinhalb Reihen

t6 + blickt P4 ins Gesicht

07 T5 scho + !WIE! der zwoa-a-hoib rEIhen.

schon wieder zweieinhalb reihen

t5 -----+ blickt P4 ins Gesicht

08 T5 jo+! SU: !pa,

super

t5 + kurzer Blick zum Spielbrett und wieder zurück zu P4s Gesicht --->

09 P4 na NA;

nein nein

10 P4 des is nix bsunderes

das ist nichts Besonderes

$11 \quad$ P4 $¥(2.1) / ¥$

p4 $\quad ¥$ nimmt Gefäß mit den Steckobjekten, schüttelt es und stellt es wieder hin $/ ¥$

12 T5 ((lacht))

13 P4 <<lächelnd > dass $\mathrm{i}$ des immer do $+_{\mathrm{p} 4}$ EInisteck $>,+{ }_{\mathrm{p} 4}$ dass ich das immer da hineinstecke

p4

+ blickt in Richtung von T6

+ blickt auf die Füße--->

$14 \mathrm{T5}+_{\mathrm{t} 6} \mathrm{na}+_{\mathrm{t} 5} \mathrm{JO}$,

na ja

t6 + blickt auf den Boden (gleiche Richtung wie P4 ----------------->>

t5 + blickt auf Hüfte von P4 ---------------------------(bis

Z.16)->

15 T5 da brAUcht $+¥$ ma (.) musst stabil STEhen-

da braucht man (.) da musst du stabil stehen

p4 -------> + Suchbewegungen zu beiden Seiten mit Blick und Kopf --->>

p4 $¥$ rutscht auf der Liege leicht hin und her---------------> 
16 T5 und + oa hond / $¥$ muass FREI werden ja?

und eine Hand muss frei werden ja?

+ blickt P4 ins Gesicht

p4

17 T5

des + isch nIt so EINfach.

das ist nicht so einfach

t5

+ blickt auf den Boden, gleiche Richtung wie P4 und T6

18 T6 $\mathrm{hm} \mathrm{hm}$

19 T5 wenn ma $\mu$ sich $¥$ überlEgt=gö=vor $/ ¥$ zwEI wOchen (Name P4), wenn man sich überlegt vor zwei Wochen, (Name von $P$ )

t5 $\mu$ stabilisiert mit RH 1.Knie von P4, mit LH die $r$. Hüfte von P4->>

t5

$¥$ beugt sich nach vorne und unten $/ ¥$

20 P4 jo (-) hot ma $¥$ des no NIT / $¥$ zsAmmbrocht=gö?

ja, hat man das noch nicht zusammengebracht, gell?

$¥$ macht einen Schritt zurück, beugt sich nach vorne /¥

Die Patientin muss zum wiederholten Mal längere Zeit stehen und gleichzeitig eine Übung mit einem Holzsteckspiel durchführen. Bereits bei der vorhergehenden Wiederholung hat sie ihr Unverständnis über den Sinn der Übung ausgedrückt. Die Therapeutinnen finden die Leistung, zweieinhalb Reihen geschafft zu haben, jedoch sehr gut und drücken explizit ihr Lob aus, prosodisch mit starken Akzentuierungen hervorgehoben (Z. 03-08) und begleitet von Blicken in das Gesicht der Patientin. Mimisch zeigt die Patientin mit einem leicht nach unten gezogenen Mundwinkel an, dass sie dies anders sieht, und verdeutlicht das in der Folge nahezu defätistisch: durch eine abwertende Geste in Z. 11, bei der sie die Schachtel mit den Holzstückchen schüttelt, durch eine begleitende und darüber hinausgehende verbale Abwertung der Übung an sich und ihres Könnens (Z. 09-13) sowie durch das Suchen nach Bestätigung mit der kurzen Aufnahme von Blickkontakt (Z. 13), nachdem sie diesem während des Lobs ausgewichen war. Die Physiotherapeutin kündigt zunächst die gegenläufige Zweite Bewertung (laut Auer \& Uhmann 1982) typischerweise mit einer Partikel an (Z. 14), bevor sie in Z. 15-17 sowohl den physiotherapeutischen Sinn als auch die erbrachte Leistung in ein positiveres Licht rückt. Der Widerspruch wird zwar mit verallgemeinerndem man abgeschwächt, es handelt sich jedoch um die stärkste Form des Widerspruchs nach Spranz-Fogasy (1986: 38), die Gegenbehauptung mit einem starken Abschlusssignal (gö?, in Z. 20). Die Patientin weicht dem direkten Blickkontakt mit den Therapeutinnen aus (ab Z. 17) und richtet sich körperlich auf der Liege und vor dem Pult anders ein. Ohne diese körperliche Abwendung von den Therapeutinnen aufzugeben, stimmt sie je- 
doch schließlich in Z. 20 in das in Z. 19 eingeleitete und von der Therapeutin prosodisch und durch die Formulierung offen gelassene Angebot einer abschließenden positiven Bewertung ein, indem das Geschaffte mit den Leistungen in früheren Therapieeinheiten verglichen wird. Auf diese Weise wird ein gemeinsames Verständnis über den Sinn und Erfolg der therapeutischen Interventionen erzielt.

\subsection{Epistemische Rechte und Pflichten im Konflikt}

Die ,künstliche Reziprozität‘ einer Therapiesituation (Pawelczyk 2011: 55 für den Kontext Psychotherapie) und die ungleiche Machtverteilung sind jedoch auch mögliche Ursachen für Konflikte, zumal Therapeut`innen professionelle Distanz und Empathie jonglieren und in ihrer Verantwortung für die Patient*innen Compliance/Adherence sicherstellen müssen. Nicht alle diese Einflussfaktoren auf den therapeutischen Prozess lassen sich in einer interaktionalen Studie rekonstruieren. In den bisherigen Beispielen haben die Patientinnen die alternative Evaluation des Therapeuten bzw. der Therapeutin übernommen. In Beispiel 4 liegt der Fall anders, hier kommt es zu einer nicht nur hinsichtlich der Lautstärke intensiven Störung der therapeutischen Beziehung.

Beispiel 4: Du hast ja ka Parese

Einheit 006, ((00:26:24.088 - 00:27:21.200))

T8 $=$ Therapeutin, $\mathrm{P} 6=$ Patientin

((P6 liegt in Seitenlage auf der Liege. T8 und P6 arbeiten bereits seit einigen Minuten daran, dass der richtige Muskel beim Heben des Knies anspringt.))

01 P6 > $¥_{\text {t8 }}$ na oba $\mathrm{i}$ ! KUNN! des [net;

nein aber ich kann das nicht

t8 >¥ holt Hocker an die Liege heran und richtet die Position ein --->

$02 \quad$ T8

[nein $¥<<$ cresc > jetzt konzenTRIER * dich >

mal $=/$ *

nein jetzt konzentrier dich mal

t8

* schüttelt

den Kopf /*

03 T8 $<<f f>=$ na! TÜR! lich kannst $¥$ du dAs > -

natürlich kannst du das 




((Genaue Anweisungen zur richtigen Ansteuerung der Muskeln zum Heben des Knies mit taktiler Unterstützung, $22 \mathrm{sec}$.))

28 P6 $<<p>i$ ! KUNN! des $\downarrow$ nEt (Name von T8). > ich kann das nicht (Name von T8)

29 T8 $>\mu(($ seufzt $)) \Delta(0.1) *(0.2) / \Delta+(0.5) / *$

t8 $>\mu$ fixiert mit LH Hüfte und mit rechter Hand Knie von P6 ---------->>

t8 $\Delta$ beugt sich mit dem Oberkörper nach vorne zu P6 / $\Delta$

* rollt mit den Augen /*

+ blickt P6 ins Gesicht --------->>

$30 \quad$ T8 tu $¥ \mathrm{a}+\mathrm{mal}(0.3)$

tu einmal

31 P6 ja i WILLS [ja]،

ja ich wills ja

32 T8 [pro]BIER a/mal $\uparrow$ NUR (0.4)

probier mal nur

33 P6 JA?

ja?

$34(0.4)$

35 T8 das knIE * LEICHT / * machen Ohne bewEgung;

das knie leicht machen ohne bewegung

t8 *schüttelt leicht den Kopf /*

36 P6 $<<$ h $>$ ! LEICHT!? >

leicht

37 T8 ge*NAU; /*

genau

t8 * nickt /*

Dieses Beispiel umfasst mehrere Abschnitte. Nach einer bereits ziemlich langen Phase des Misslingens der Bewegungsausführung zeigen sich die Patientin und die Therapeutin prosodisch, mimisch und gestisch frustriert. Auf die Rechtfertigung der Patientin in Z. 01, dass sie die angeforderte Bewegung schlicht nicht ausführen könne, folgt ein sehr gesichtsbedrohender Angriff der Therapeutin (Z. 02-04) in großer Lautstärke, mit starken Akzentuierungen und begleitet von 
einer wegwerfenden Handbewegung, dass das Problem bei der mangelnden Konzentration liegen müsse, da der anzusteuernde Muskel nicht gelähmt sei.

Epistemisch und deontisch betrachtet kann die Therapeutin die Ehrlichkeit des Bemühens der Patientin infrage stellen und mehr Anstrengung einfordern. Es handelt sich dabei aber um einen Angriff auf das positive ebenso wie auf das negative Gesicht. Im Therapiekontext ist compliance nach Anweisungen präferiert bzw. auch verzögerte compliance markiert (vgl. Kent 2012 für eine Diskussion von incipient compliance). Andererseits sind die Therapeut*innen Dienstleister ${ }^{\star}$ innen, die auf die Mitarbeit der Patient*innen angewiesen und der partizipativen Entscheidungsfindung verpflichtet sind, also den Patient*innen bei der Bewältigung der alltagsrelevanten Therapieziele helfen (vgl. Frommelt \& Grötzbach 2010). Nach dieser kurzen Eskalation versucht es die Therapeutin mit einer neuerlichen Anleitung. In Z. 28 bekräftigt die Patientin ihre Unfähigkeit leise, aber eindeutig und mit einer direkten Anrede der Therapeutin. Diese quittiert es mit einem Augenrollen, das die Patientin aufgrund ihrer Position nicht sehen kann, während das Seufzen für sie wahrnehmbar ist. Es folgt eine neuerliche Aushandlung der geforderten Bewegung (Z. 30-37), wiederum mit manueller Unterstützung durch die Therapeutin und in harmonischerer Art, sowohl was die Lautstärke und Intensität als auch die Resonanz zwischen Therapeutin und Patientin als Anzeichen für Intersubjektivität (vgl. Kärkkäinen 2006) angeht.

Dieses durch starke emotionale Erregung gekennzeichnete Beispiel stellt eine Ausnahme im Korpus dar. Es ist zwar häufig so, dass Anleitungen bei wiederholten Durchgängen prosodisch und durch stärkeren Druck bei der manuellen Unterstützung intensiviert werden, ebenso wie das Lob nach letztendlichem Gelingen, auch kommt es zeitweise zu kleinen Meinungsverschiedenheiten über die Bewertung des Erreichten (siehe Beispiel 3), doch ein offener Konflikt wie in Beispiel 4 ist selten. Es wird jedoch wieder schnell zur Refokussierung übergegangen - der Widerstand der Patientin vermittelt der Therapeutin, dass eine andere Anleitungsstrategie erforderlich ist, was am Abschluss der Bearbeitungssequenz (mehrere Minuten nach Ende des Transkriptausschnitts) zu einem einigermaßen zufriedenstellenden Ergebnis für beide Beteiligten führt.

Quasi das Gegenstück zu diesem Typ stellt das implizite Widersprechen durch das Nicht-Eingehen auf patientenseitiges troubles telling dar. Diese Form kann hier aus Gründen des Umfangs nicht anhand eines Beispiels diskutiert werden. Werden Bewertungen durch die Therapeut*innen verweigert, ist dies meist kein Zeichen für mangelnde Empathie oder Vermeidungsverhalten, sondern eine Strategie, die Aufmerksamkeit der Patient*innen von den Defiziten abzuziehen (vgl. Parry 2004b: 1002). Dieses Nicht-Eingehen auf negative Emotionalität und der Fokus auf Urteile über Fähigkeiten und die Ergebnisse der Therapie wird etwa von Josephson et al. (2015: 134-135) allerdings kritisch gesehen. 


\section{Zusammenfassung}

Abstrahiert man aus den identifizierten Sequenzen des Nicht-Übereinstimmens Muster der sequenziellen Organisation und der multimodalen Realisierung, ergeben sich drei obligatorische Schritte und einige Tendenzen hinsichtlich der interaktionalen Aushandlung.

Initiierung (z. B. Beispiel 3, Z. 09-13): Die betrachteten Sequenzen werden fast ausschließlich durch eine negative Selbstwahrnehmung (Körperempfindung) der Patientinnen und Patienten angestoßen, und zwar nicht am Beginn einer Übung, sondern nach bereits mehrfachen Versuchen, eine Bewegung auszuführen, oder gegen Ende einer längeren Übungsphase. Die Selbstwahrnehmung wird teils nur mit Unmut signalisierenden Vokalisationen, teils verbal mit prosodischer Intensivierung oder durch körperliche Displays von Unzufriedenheit (z. B. Unterbrechen der Übung) angezeigt. In allen Fällen wird eine Reaktion der Therapeutinnen und Therapeuten konditionell relevant gesetzt.

Widerspruch (z. B. Beispiel 2, Z. 06-08): Der Widerspruch von therapeutischer Seite kann sehr schnell erfolgen, ihm geht jedoch häufig eine kurze Phase mit einem verkörperten Display der Prüfung der negativen Bewertung voraus (z. B. mit haptischer Wahrnehmung, oft verbunden mit einer Veränderung der körperlichen Positionierung). Die Realisierung des Widerspruchs erfolgt meistens in expliziter verbaler Form, selten aber auch implizit durch körperliches Handeln (Weitermachen) oder multimodale Displays wie Kopfschütteln. Verbaler Widerspruch beinhaltet ein alternatives, meist nicht gesichtsbedrohendes Erklärungsmodell für die aufgetretenen Schwierigkeiten. Erklärungsmodelle beziehen sich hauptsächlich auf Aspekte, die außerhalb der Verantwortung der Patient*innen liegen, oder enthalten eine Abschwächung der von den Patient*innen beobachteten Einschränkungen.

Weitere Aushandlungsprozesse (z. B. Bsp. 4, Z. 07-11): Der Widerspruch wird im weiteren Verlauf nicht immer aufgelöst. Eine Möglichkeit ist die Fortsetzung und Vertiefung der Erklärungen durch die Therapeut*innen, was insbesondere dann geschieht, wenn die alternative, nicht-abwertende Erklärung für Probleme verbal oder körperlich zurückgewiesen wird oder die Problembekundung wiederholt wird. In diesen Fällen wird das Problem in mehreren Schleifen bearbeitet.

Wie die Beispiele zeigen, ist Nicht-Übereinstimmen keineswegs ein Anzeichen für Konflikte zwischen den Interagierenden. Auch beim konfliktträchtigen, emotionalen Divergieren in Beispiel 4 gelangen Therapeutin und Patientin nach einer Phase der beiderseitigen Irritation letztendlich $\mathrm{zu}$ einer geteilten Evaluation und neuerlichen Aufgabenorientierung. Im Sinne der hier vorgenommenen Analysen kann man davon ausgehen, dass therapeutenseitiger, verbal und/ 
oder multimodal vollzogener Widerspruch in den meisten Fällen ein nur vorläufiges Auseinandergehen im therapeutischen Prozess ist, das einen notwendigen Zwischenschritt darstellt, um die gemeinsamen Aufgaben zu erfüllen: die aktuelle Bewegungsausführung oder die Übung zu bewältigen, Schmerzen zu vermeiden und langfristige Erfolge anzupeilen.

\section{Transkriptionskonventionen ${ }^{3}$}

\begin{tabular}{|c|c|}
\hline T01 & Teilnehmer*innenkürzel verbal \\
\hline t01 & Teilnehmer*innenkürzel verkörperte Aktivität \\
\hline /* & Gestik (einschließlich gestische Kopfbewegungen) \\
\hline & $\begin{array}{l}\text { ( }{ }^{*} \text { Einsatz oder Änderung einer verkörperten Aktivität, } /{ }^{*}=\text { Schlusspunkt) } \\
\text { manuelles therapeutisches Handeln }\end{array}$ \\
\hline+ & Blick \\
\hline $\int$ & Berührung \\
\hline$¥$ & Körperbewegung und Objekthandeln \\
\hline$\Delta$ & Veränderung in der Körperorientierung und Proxemik \\
\hline$>+$ & Display beginnt vor dem Transkriptausschnitt \\
\hline$>\mid-->>$ & Display geht über die Zeile / über den Transkriptausschnitt hinaus \\
\hline $\begin{array}{l}\text {, } \mathrm{RH}, \mathrm{BH} \text {, } \\
\mathrm{RL}, \mathrm{BL}\end{array}$ & linke Hand, rechte Hand, beide Hände, linkes Bein, rechtes Bein, beide Beine \\
\hline
\end{tabular}

\section{Literatur}

Auer, J. C. P. \& Susanne Uhmann (1982): Aspekte der konversationellen Organisation von Bewertungen. Deutsche Sprache 10, 1-32.

Clark, Herbert H. \& Susan Brennan (1991): Grounding in communication. In Lauren Resnick, John M. Levine \& Stephanie D. Teasley (Hrsg.), Perspectives on socially shared cognition, 127-149. Washington: American Psychological Association.

Deppermann, Arnulf (2013): Multimodal interaction from a conversation analytic perspective. Journal of Pragmatics 46 (1), 1-7.

Deppermann, Arnulf \& Reinhold Schmitt (2008): Verstehensdokumentationen: Zur Phänomenologie von Verstehen in der Interaktion. Deutsche Sprache 36 (3), 220-245.

Fries, Wolfgang \& Susanna Freivogel (2010): Motorische Rehabilitation. In Peter Frommelt \& Hubert Lösslein (Hrsg.), Neuro-Rehabilitation. Ein Praxishandbuch für interdisziplinäre Teams, 225-266. Berlin u. a.: Springer.

3 An dieser Stelle werden nur jene Transkriptionszeichen angegeben, die über GAT 2 hinausgehen. 
Frommelt, Peter, Holger Grötzbach (2010): Kontextsensitive Neurorehabilitation: Einführung in die klinische Neurorehabilitation. In Peter Frommelt, Hubert Lösslein (Hrsg.), NeuroRehabilitation. Ein Praxishandbuch für interdisziplinäre Teams, 3-22. Berlin u. a.: Springer.

Gill, Virginia Teas (1998): Doing attributions in medical interaction: Patients' explanations for illness and doctors' responses. Social Psychology Quarterly 61 (4), 342-360.

Goodwin, Charles (1994): Professional vision. American Anthropologist 96 (3), 606-633.

Goodwin, Charles \& Marjorie Harness Goodwin (1992): Assessments and the construction of context. In Alessandro Duranti \& Charles Goodwin (Hrsg.), Retinking context, 147-190. Cambridge: Cambridge University Press.

Heidtmann, Daniela \& Marie-Joan Föh (2007): Verbale Abstinenz als Form interaktiver Beteiligung. In Reinhold Schmitt (Hrsg.), Koordination. Analysen zur multimodalen Interaktion (Studien zur Deutschen Sprache 38), 263-292. Tübingen: Narr.

Heritage, John (2002): Oh-prefaced responses to assessments: a method of modifying agreement/disagreement. In Cecilia E. Ford, Barbara A. Fox \& Sandra A. Thompson (Hrsg.), The language of turn and sequence (Oxford studies in sociolinguistics), 196-224. Oxford u. a.: Oxford University Press.

Heritage, John (2012): The epistemic engine: Sequence organization and territories of knowledge. Research on Language and Social Interaction 45 (1), 30-52.

Heritage, John \& Geoffrey Raymond (2005): The terms of agreement: Indexing epistemic authority and subordination on talk-in-interaction. Social Psychology Quarterly 68 (1), 15-38.

Josephson, Iréne, Robyn Woodward-Kron, Clare Delany \& Amy Hiller (2015): Evaluative language in physiotherapy practice. How does it contribute to the therapeutic relationship? Social Science and Medicine 143, 128-136.

Kärkkäinen, Elise (2006): Stance taking in conversation: From subjectivity to intersubjectivity. Text and Talk 26 (6), 699-731.

Kent, Alexandra (2012): Compliance, resistance and incipient compliance when responding to directives. Discourse Studies 14 (6), 711-730.

Lee, Seung-Hee \& Hiroko Tanaka (2016): Affiliation and alignment in responding actions. Journal of Pragmatics 100, 1-7.

Levinson, Stephen C. (2000): Pragmatik, 3. Aufl. Tübingen: Niemeyer.

Martin, James Robert \& Peter R.R. White (2005): The language of evaluation. Appraisal in English. Basingstoke, New York: Palgrave Macmillan.

Mondada, Lorenza (2013): Interactional space and the study of embodied talk-in-interaction. In Peter Auer, Martin Hilpert, Anja Stukenbrock \& Benedikt Szmrecsanyi (Hrsg.), Space in language and linguistics. Geographical, interactional and cognitive perspectives (Linguae \& litterae 24), 247-275. Berlin, New York: De Gruyter.

Mondada, Lorenza (2014): Conventions for multimodal transcriptions. Abrufbar unter URL: https://mainly.sciencesconf.org/conference/mainly/pages/Mondada2013_conv_multi modality_copie.pdf (letzter Zugriff 10.02.2018).

Parry, Ruth H. (2004a): Communication during goal-setting in physiotherapy treatment sessions. Clinical Rehabilitation 18, 668-682.

Parry, Ruth Helen (2004b): The interactional management of patients' physical incompetence: a conversation analytic study of physiotherapy interactions. Sociology of Health and Illness 26 (7), 976-1007. 
Pawelczyk, Joanna (2011): Talk as therapy. Psychotherapy in a linguistic perspective. Berlin, Boston: De Gruyter.

Peräkylä, Anssi \& Sanna Vehviläinen (2003): Conversation analysis and the professional stocks of interactional knowlege. Discourse and Society 14 (6), 727-750.

Pomerantz, Anita (1984): Agreeing and disagreeing with assessments: Some features of preferred/dispreffered turn shapes. In J. Maxwell Atkinson \& John Heritage (Hrsg.), Structures of social action (Studies in emotion and social interaction), 57-101. Cambridge u. a.: Cambridge University Press.

Ruusuvuori, Johanna (2007): Managing affect: integration of empathy and problem solving in health care encounters. Discourse Studies 9 (5), 597-622.

Schegloff, Emanuel A. (1972): Notes on conversational practice: formulating place. In David N. Sudnow (Hrsg.), Studies in social interaction, 75-119. New York u. a.: The Free Press.

Schegloff, Emanuel A. (2007): Sequence organization in interaction. A primer in Conversation Analysis I. Cambridge u. a.: Cambridge University Press.

Schmitt, Reinhold (Hrsg.) (2007): Koordination. Analysen zur multimodalen Interaktion (Studien zur Deutschen Sprache 38). Tübingen: Narr.

Selting, Margret, Peter Auer, Dagmar Barth-Weingarten, Jörg Bergmann, Pia Bergmann, Karin Birkner, Elisabeth Couper-Kuhlen, Arnulf Deppermann, Peter Gilles, Susanne Günthner, Martin Hartung, Friederike Kern, Christine Mertzlufft, Christian Meyer, Miriam Morek, Frank Oberzaucher, Jörg Peters, Uta Quasthoff, Wilfried Schütte, Anja Stukenbrock \& Susanne Uhmann (2009): Gesprächsanalytisches Transkriptionssystem 2 (GAT 2). Gesprächsforschung. Online-Zeitschrift zur verbalen Interaktion 10, 353-402.

Spranz-Fogasy, Thomas (1986): , widersprechen'. Zu Form und Funktion eines Aktivitätstyps in Schlichtungsgesprächen. Eine gesprächsanalytische Untersuchung. Tübingen: Narr.

Stevanovic, Melisa \& Anssi Peräkylä (2012): Deotic authority in interaction. The right to announce, propose, and deside. Research on Language and Social Interaction 45 (3), 297-321. 
